\title{
Effects of peeling and steam-heating treatment on mechanical properties and dimensional stability of oriented Phyllostachys makinoi and Phyllostachys pubescens scrimber boards
}

\author{
Min Jay Chung ${ }^{1}$. Sheng Yang Wang ${ }^{2,3}$
}

Received: 7 December 2017 / Accepted: 10 April 2018 / Published online: 29 May 2018

(C) The Japan Wood Research Society 2018

\begin{abstract}
Epidermal peeling treatment (EPT) and steam-heating treatment (SHT) are two popular pretreatments for bamboo processing. This study examined the effects of EPT and SHT on strength properties, profile density distribution, internal bond strength (IB), rate of springback, nail withdrawal resistance, and dimensional stability of oriented bamboo scrimber board (OBSB) made of moso bamboo (Phyllostachys pubescens Mazel) and makino bamboo (P. makinoi Hayata) strips. Results obtained using non-destructive testing (NDT) revealed that EPT for moso bamboo in the processing of OBSB caused lower ultrasonicwave velocity $\left(V_{\mathrm{u}}\right)$ and dynamic modulus of elasticity $\left(\mathrm{DMOE}_{\mathrm{u}}\right)$ parallel to the fiber direction, but higher $V_{\mathrm{u}}$ and $\mathrm{DMOE}_{\mathrm{u}}$ perpendicular to the fiber direction. However, EPT slightly affected variations in modulus of elasticity (MOE) and modulus of rupture (MOR) of moso bamboo. In contrast, the effects of SHT on $V_{\mathrm{u}}$ and $\mathrm{DMOE}_{\mathrm{u}}$ were inconsistent and insignificant among the OBSB samples. On the other hands, SHT caused increasing in MOE and MOR of OBSB, but leads to decrease in MOE and MOR of OBSB comprising bamboo strips after EPT. Both EPT and SHT contributed to more uniform profile densities in OBSB and had a positive impact on nail withdrawal resistance. EPT increased IB of moso bamboo and SHT enhanced IB of makino bamboo with epidermis only. Bamboo strips after SHT resulted in significant decrease in water absorption of all OBSB specimens. Reduction in swelling as a result of SHT not only improved the dimensional stability of OBSB but also enhanced strength.
\end{abstract}

Keywords Bamboo culms $\cdot$ Oriented bamboo scrimber board $\cdot$ Mechanical properties $\cdot$ Dimensional stability

\section{Introduction}

Bamboo (Bambusoideae) is a perennial lignified plant, which distributes in tropical and subtropical regions. Taiwan is famous for richness forest resource, where many bamboo species widely distribute from the coastlines to the mountains. Makino bamboo (Phyllostachys makinoi Hayata) and moso bamboo (P. pubescens Mazel) are two

Sheng Yang Wang

taiwanfir@dragon.nchu.edu.tw

1 Experimental Forest, National Taiwan University, No. 12, Section 1, Chien-Shan Road, Chu-Shan, Nantou Hsien 55750, Taiwan, Republic of China

2 Department of Forestry, National Chung-Hsing University, No. 250, Kou Kung Road, Taichung 402, Taiwan, Republic of China

3 Agricultural Biotechnology Research Center, Academia Sinica, Taipei 128, Taiwan, Republic of China main economic bamboos in Taiwan. Because bamboo possesses many advanced properties, such as fast growth, easily available, renewable naturally, as well as excellent mechanical and strength properties comparable to that of timber, it has become one of the most important nontimber forest products in Taiwan and other Asian countries [1-3]. Also, more and more attentions have been paid to the development and utilization of laminated bamboo material recently [2, 4-9]. Although laminated bamboos posses the multiple application, the processing of bamboo to fit the standardized production conditions would lose some of its unique properties and increasing the cost of production. In recent years, oriented bamboo scrimber (OBS), an engineered composite made of parallel bamboo bundles [10], is increasing research interest [10-14]. Mechanical properties of beams or boards manufactured by OBS have been found to be similar to or surpass those of timber or timber-based products [13, 14]. Compared with laminated bamboo, OBS is superior in that it has 
higher slenderness ratio, higher raw material utilization rate [10] and lower production cost.

Structural composite products made of bamboo have low bonding strength and rough structure defects [11, 15]; however, due to recent advances in manufacturing equipment, adhesives, and hot pressing technology, OBS enhanced composite properties, including good surface texture, higher hardness, and higher longitudinal strength [12]. Hence, OBS has been widely applied to make railing, flooring, furniture, and applied to construction and civil engineering. Undeniable, as similar to other lignocellulosic materials, bamboo's high hydrophilicity characteristics causes several disadvantages for utilization, e.g., decay, shrinkage, deformation, durability, and dimensional stability [1]. To solve the problems mentioned above, heat treatment $[16,17]$ and chemical modifications $[18,19]$ have been developed to improve the durability of woody materials. Heat-treated woody materials would cause hemicellulose degradation, lignin softening, and reduced hydrophilicity of cellulose [16]; however, increasing hydrophobicity of lignocellulosic materials by hightemperature processing will enhance both natural dimensional stability and durability [17, 20, 21]. Also, Yildiz et al. reported heat treatment improved surface properties, durability, and mechanical properties in engineered bamboo composites [22]. Furthermore, their results indicated whether steam-heating procedure incorporated in the manufacturing of oriented bamboo scrimber board (OBSB) would enhance both its properties and applicability.

Density, internal bond strength (IB), modulus of elasticity (MOE), and modulus of rupture (MOR) are common indicators for bamboo strength [23]. Non-destructive techniques (NDT) have been widely conducted to evaluate the strength assessment of wood products. Ross and his coworkers found a good correlation between MOE predicted by the acoustic wave and the mean lumber MOE by determining the longitudinal speed of stress wave transmission [24]. Lin et al. [25] and Lee et al. [9] also applied NDT to evaluate the quality of moso bamboo lamina and laminated bamboo flooring; the results revealed close relationships between dynamic modulus of elasticity (DMOE), MOE, and MOR, and a positive correlation between DMOE and MOR $\left(R^{2}=0.92, p>0.01\right)$. Moreover, the NDT using ultrasonic wave in these two studies proved to be useful in evaluating the mechanical strength of laminated bamboo materials.

In this study, the effects of peeling and steam-heating on OBSB's strength properties, profile density distribution, IB, rate of springback, nail withdrawal resistance and dimensional stability were examined using NDT. The testing OBSBs were made of makino bamboo and moso bamboo with either epidermis removed or kept intact. Our results not only contribute to more thorough understanding of properties of peeled and steam-heated bamboo but also provide expedient information for development and applications of bamboo-based engineering materials.

\section{Materials and methods}

\section{Oriented bamboo scrimber board (OBSB)}

Three-year-old moso bamboo and makino bamboo culms were collected from the Experimental Forest of National Taiwan University in Nan-Tou County, Taiwan, in October 2015. Also, the same age of bamboo samples was also collected from Lin' an County, Zhejiang Province, China. In addition to makino bamboo and moso bamboo culms were collected from Taiwan and China, respectively. Moso bamboo culms collected from Taiwan samples were divided into two groups, i.e., unpeeled/with the epidermis and peeled/without epidermis. All samples were pre-treated with an alkaline solution containing $2 \%$ potassium hydroxide $(\mathrm{KOH})$ at $100{ }^{\circ} \mathrm{C}$ for $30 \mathrm{~min}$ and then oven-dried at $80^{\circ} \mathrm{C}$. For steam-heating treatment (SHT), bamboo strips were placed in a steam-heating furnace at $120^{\circ} \mathrm{C}$ for $6 \mathrm{~h}$.

After pretreatment, bamboo culms were extruded into $450 \times 1.0-2.0 \mathrm{~mm}$ (length $\times$ width) of thin strips by mechanical processing. Then they were placed unidirectionally in a $450 \times 450 \times 12.0 \mathrm{~mm}$ (length $\times$ width $\times$ thickness) of the iron frame to form a board at $0.90 \mathrm{~g} / \mathrm{cm}^{3}$ density. The adhesive used in this study is a water-soluble urea formaldehyde (UF) resin with a $63.6 \%$ solid content provided by Wood Glue Industrial Co., Tainan, which was applied at $10 \%$ based on raw materials. The strips were hot-pressed under curing temperature of $120^{\circ} \mathrm{C}$ at $147 \mathrm{~Pa}$ for $12 \mathrm{~min}$, followed by 10 -min cooling. Figure 1 illustrates the manufacturing flowchart of OBSB from bamboo strips.

Before the experiments, all specimens were conditioned in a controlled environment with the temperature at $20{ }^{\circ} \mathrm{C}$ and relative humidity (RH) at $65 \%$ for 2 weeks. Table 1 summarizes the codes and treatments of the eight experimental OBSB specimens $(n=9)$ in this study.

\section{Non-destructive evaluation}

Non-destructive evaluation techniques were conducted to evaluate the ultrasonic-wave velocity $\left(V_{\mathrm{u}}\right)$ and DMOE using a portable ultrasonic non-destructive testing device (Sylvatest Duo, Saint Sulpice, Switzerland) at a frequency of $22 \mathrm{kHz}$. Specimens were placed between the transmitting and receiving transducers $(n=9)$, and the travel time of the ultrasonic wave (transmission time) were recorded. 


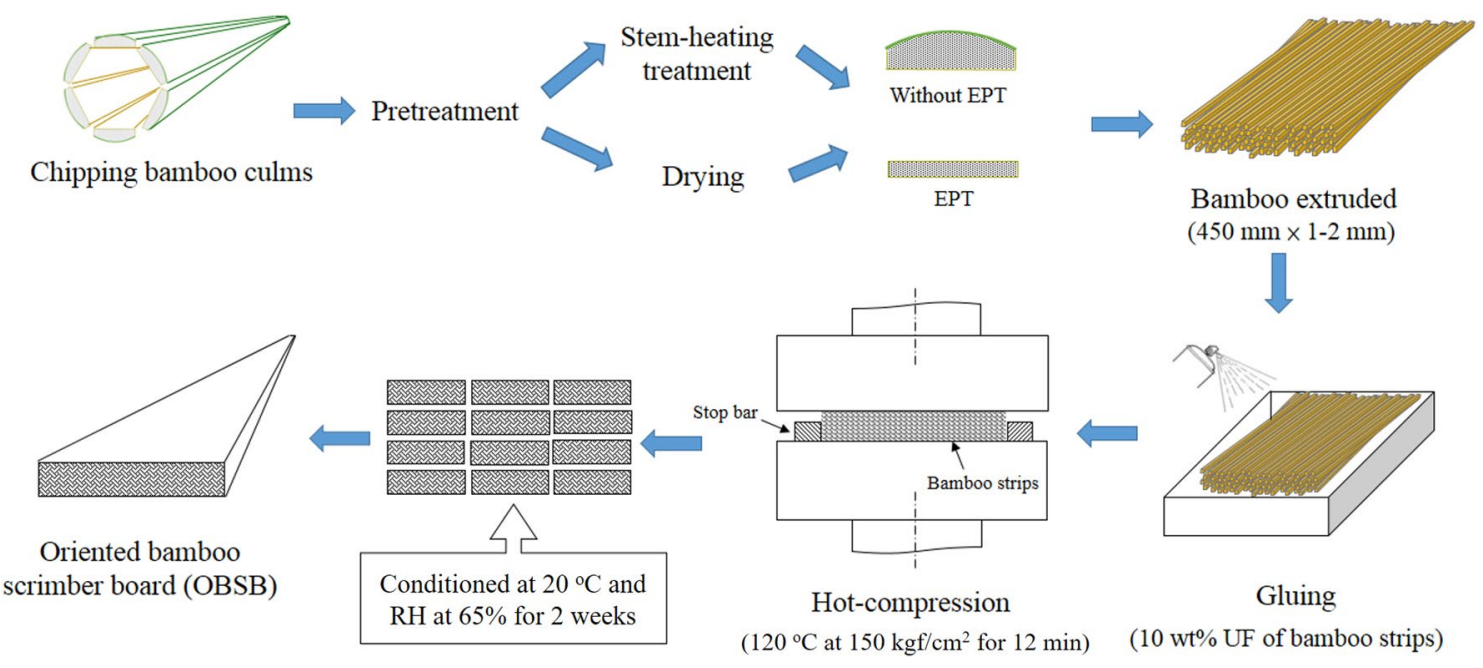

Fig. 1 Manufacturing process of oriented bamboo scrimber board (OBSB)

Table 1 Codes and treatments of experimental oriented bamboo scrimber board (OBSB) specimens

\begin{tabular}{llllll}
\hline No. & Origin of Bamboos & Species & EPT & SHT & Code \\
\hline 1 & Taiwan & Makino bamboo & No & No & TPmE \\
2 & & & & Yes & TPmE-H \\
3 & & Moso bamboo & No & No & TMosoE \\
4 & & & Yes & TMosoE-H \\
5 & & & Yes & No & TMoso \\
6 & & & Yes & TMoso-H \\
7 & China & Moso bamboo & Yes & No & CMoso \\
8 & & & & Yes & CMoso-H \\
\hline
\end{tabular}

$E P T$ epidermal peeling treatment, bamboo samples were peeled with epidermis, TPmE without EPT and SHT, makino bamboo culms were collected from Taiwan, TPmE- $H$ without EPT, makino bamboo culms were collected from Taiwan, TMosoE without EPT and SHT, moso bamboo culms were collected from Taiwan, TMosoE- $H$ without EPT, moso bamboo culms were collected from Taiwan, TMoso with EPT, moso bamboo culms were collected from Taiwan, TMoso$H$ with EPT and SHT, moso bamboo culms were collected from Taiwan, CMoso With EPT, moso bamboo culms were collected from China, CMoso- $H$ with EPT and SHT, moso bamboo culms were collected from China, SHT Steam-heating treatment, bamboo strips were placed in a steam-heating furnace at $120^{\circ} \mathrm{C}$ for $6 \mathrm{~h}$

\section{Mechanical strength analysis}

The mechanical strength of OBSB specimens $(n=9)$ were examined using the American Society Testing and Materials (ASTM) method standard D-1037 [26]. The static bending test was carried out using a Shimadzu UH-10A (Tokyo, Japan) universal-type testing machine according to the center-loading method for specimens. A concentrated bending load was applied at the center with a span 15 times the thickness of the specimen. Both MOE and MOR were calculated from load-deflection curves.

\section{Profile density distribution analysis}

Figure 2 shows the schematic view of the OBSB specimen for analysis of profile density distribution $(n=9)$. The specimens were $50.0 \times 50.0 \times 12.0 \mathrm{~mm}$ (length $\times$ width $\times$ thickness), divided into two halves, with each half made up of three OBSB layers. The dimensions of each OBSB layer were $50.0 \times 50.0 \times 2.0 \mathrm{~mm}$ (length $\times$ width $\times$ thickness). The density of each layer was calculated according to its weight and volume measured after each grinding by a sand mill. All density
Fig. 2 Schematic view for profile density analysis of OBSB

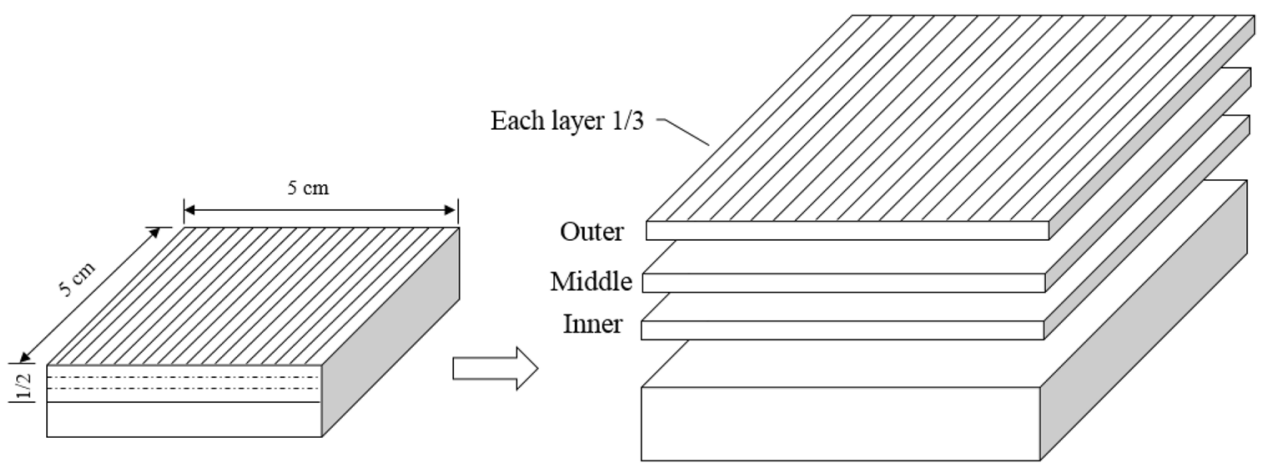


values thus obtained were plotted to illustrate the profile density distribution.

\section{Internal bond strength (IB) analysis}

The tensile strength perpendicular to the surface was determined using nine samples of $50.0 \times 50.0 \mathrm{~mm}$ from each panel according to ASTM standard D-1037 [26]. The rupture load $(P)$ was determined.

\section{Springback (SB) analysis}

Specimens of $12.0 \mathrm{~mm}$ thickness were placed in a controlled environment with $65 \%$ RH for 2 weeks. The percentage of SB was calculated $(n=9)$.

\section{Nail withdrawal resistance analysis}

According to the method of test for particleboards of the Chinese National Standards (CNS 2215 standard) [27], the OBSB specimens $(n=9)$ were placed in a controlled environment with $65 \%$ RH for 3 weeks. The dimensions of each OBSB specimen were $100 \times 50.0 \times 12.0 \mathrm{~mm}$ (length $\times$ width $\times$ thickness), and those of a wood screw was $2.7 \times 16.0 \mathrm{~mm}$ (diameter $\times$ length). Wood screws were drilled vertically into OBSB specimens to a depth of $11.0 \mathrm{~mm}$ and then pulled up vertically at $2.0 \mathrm{~mm} / \mathrm{min}$. The maximum pull loading was measured, and the average of three measurements was taken as the nail withdrawal resistance.

\section{Dimensional stability}

The OBSB specimens were tested using ASTM method standard D-1037 [26] to determine water absorption (WA), thickness swelling (TS), and volumetric swelling (S). Initial thickness in the middle of the test specimen was first measured with a micrometer. Number of specimens per variable is 9 repeats. Then all test specimens were placed in parallel $30 \mathrm{~mm}$ under water and soaked for 2 and $24 \mathrm{~h}$ before the thickness was measured again.

\section{Analysis of variance}

All multiple comparisons of physical and mechanical properties were subjected to Tukey's test and analysis of variance (ANOVA). Significant differences between mean values of control and experimental specimens $(n=9)$ were determined using the Duncan's multiple range test.

\section{Results and discussion}

\section{Ultrasonic-based dynamic properties}

\section{Ultrasonic-wave velocity $\left(V_{u}\right)$}

As shown in Table 2, the standard deviations of mean density $(\rho)$ of specimens range from 0.02 to 0.06 , indicating no significant difference in density $(p>0.05)$ among these eight OBSB samples that had been hot-pressed into $0.90 \mathrm{~g} / \mathrm{cm}^{3}$. Furthermore, the ultrasonic-wave velocities parallel to the fiber direction, $V_{\mathrm{u}(/ /)}$, were in the order of makino bamboo culms were collected from Taiwan with the epidermis and SHT (TPmE-H) $\fallingdotseq$ makino bamboo culms were collected from Taiwan without EPT and SHT $(\mathrm{TP} m \mathrm{E})>$ moso bamboo culms were collected from Taiwan without EPT and SHT (TMosoE) $\fallingdotseq$ moso bamboo culms were collected from Taiwan with the epidermis and SHT (TMosoE-H) $>$ moso bamboo culms were collected from Taiwan with EPT and SHT (TMoso-H) $\fallingdotseq$ moso bamboo culms were collected from Taiwan with EPT (TMoso) $>$ moso bamboo culms were collected from China with EPT only (CMoso) $\fallingdotseq$ moso bamboo culms were collected from China with EPT and SHT (CMoso-H). A significant difference in $V_{\mathrm{u}(/ /)}$ between OBSB made of moso bamboo with and without epidermis was observed. In other words, bamboo culms after epidermal peeling treatment (EPT) undermine ultrasonic wave transmission as evidenced by the lower $V_{\mathrm{u}(/ /)}$ of TMoso, whether SHT or not, compared with TMosoE. On the other hand, SHT had the inconsistent and insignificant influence on $V_{\mathrm{u}(/ /)}$. Meanwhile, OBSB comprised of makino bamboo had higher $V_{\mathrm{u}(/ /)}$ than by moso bamboo from both Taiwan and China. Finally, OBSB encompassed Taiwan-origin bamboo had significant higher $V_{\mathrm{u}(/ /)}$ than their China-origin samples. Lee et al. in their analysis on laminated moso bamboo flooring obtained $V_{\mathrm{u}(/ /)}$ ranging between 4016 and $4174 \mathrm{~m} / \mathrm{s}$, implying similar transmission time for both types of the bamboo-based composite board [9]. Moreover, OBSB specimens made of China-origin moso bamboo had $V_{\mathrm{u}(/ /)}$ values identical to those of laminated bamboo.

In regard to ultrasonic-wave velocity perpendicular to the fiber direction, $V_{\mathrm{u}(\perp)}$, Table 2 reveals much lower $V_{\mathrm{u}(\perp)}$ for TPmE, TMosoE, TMoso and, CMoso, only 23.1, 27.3, 36.8 , and $36.8 \%$, respectively. However, the $V_{\mathrm{u}(/ /)}$ was more significant than $V_{\mathrm{u}(\perp)}$ was because OBSB, which was a composite with reorganized the fiber network, had less influence on ultrasonic wave transmitted parallel to the fiber direction. In contrast, transmission of ultrasonic wave perpendicular to fiber direction would be slowed down in the absence of horizontal fiber structure with small gaps in between the fine bamboo strips. Without 


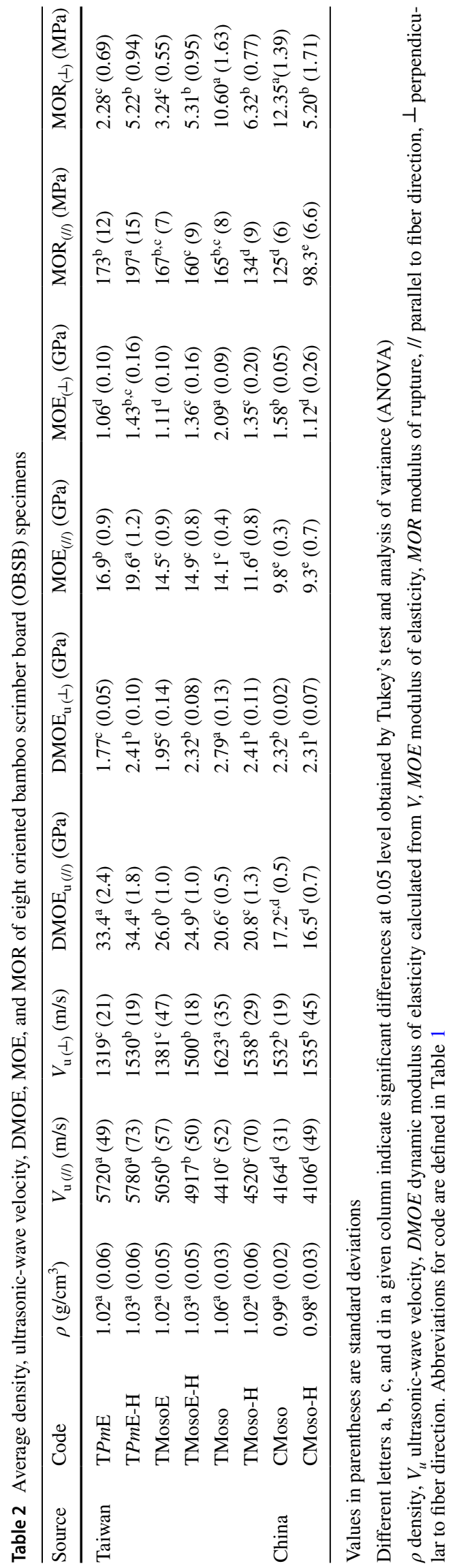

SHT specimens after EPT had higher $V_{\mathrm{u}}\left({ }^{\perp}\right)$ than those with epidermis intact, indicating EPT might have a positive effect on $V_{\mathrm{u}\left(\perp^{\perp}\right)}$. In general, all specimens after SHT, except TMoso, had higher $V_{\mathrm{u}(\perp)}$ compared with their nonheated counterparts. It indicated that SHT contributes to enhancing $V_{\mathrm{u}(\perp)}$. Compared with $V_{\mathrm{u}(\perp)}$ of $1710 \mathrm{~m} / \mathrm{s}$ for laminated moso bamboo flooring reported by Lee et al., the OBSB specimens had comparable transmission time, again indicating similarity in both types of the bamboobased composite board [9].

\section{Dynamic modulus of elasticity (DMOE)}

Table 2 demonstrates the $\mathrm{DMOE}_{\mathrm{u}(/ /)}$ values of OBSB specimens comprising bamboo without SHT were in the order of $\mathrm{T} P m \mathrm{E}>\mathrm{TMosoE}>\mathrm{TMoso}>\mathrm{CMoso}$ with significant difference $(p<0.05)$. Again, OBSB made with bamboo culms after EPT whether SHT or not, had lower $\mathrm{DMOE}_{\mathrm{u}(/ /)}$ while the effect of SHT on $\mathrm{DMOE}_{\mathrm{u}(/ /)}$ was inconsistent and insignificant. OBSB made of makino bamboo had significant higher $\mathrm{DMOE}_{\mathrm{u}(/)}$ than moso bamboo groups from both Taiwan and China. OBSB made of Taiwan-origin bamboo had significant higher $\mathrm{DMOE}_{\mathrm{u}(/ /)}$ than made of China-origin bamboos. Lee et al. obtained a $\mathrm{DMOE}_{\mathrm{u}(/ /)}$ of $10.4 \mathrm{GPa}$ for Taiwan-origin moso bamboo laminated board [9], which is much lower than OBSB observed in this study. The difference between two studies might due to the thermal compression process in the manufacture of OBSB. Hot pressing contributed to increase density, leading to higher DMOE. Comparatively, $\mathrm{DMOE}_{\mathrm{u}(\perp)}$ of all eight specimens were much lower than their $\mathrm{DMOE}_{\mathrm{u}(/ /)}$. Higher $\mathrm{DMOE}_{\mathrm{u}(\perp)}$ was observed in OBSB comprising bamboo strips after EPT, indicating positive impact of EPT on $\mathrm{DMOE}_{\mathrm{u}(\perp)}$. On the other hand, SHT increased $\mathrm{DMOE}_{\mathrm{u}(\perp)}$ in OBSB comprising unpeeled bamboo while decrease in $\mathrm{DMOE}_{\mathrm{u}(\perp)}$ was observed in OBSB made using steam-heated bamboo without epidermis.

\section{Mechanical strength analysis}

\section{Modulus of elasticity (MOE)}

Table 2 shows that $\mathrm{MOE}_{(/ /)}$of OBSB specimens made of without SHT bamboo was in the order of TPmE $>$ TMosoE $=$ TMoso $>$ CMoso. The $\mathrm{MOE}_{(/ /)}$of makino bamboo with epidermis intact was significantly higher than those of moso bamboo from both Taiwan and China. Chung and Wang had obtained the similar results for untreated makino and moso bamboo specimens [28]. Moreover, OBSB specimens made of Taiwan-origin bamboo had markedly higher $\mathrm{MOE}_{(/ /)}$than those made of China-origin bamboo $(p<0.05)$.

The same trend of $\mathrm{MOE}_{(/ /)}$was observed, which is TP $m$ E-H $>$ TMosoE-H $>$ TMoso-H $>$ CMoso-H for OBSB specimens comprising steam-heated bamboo strips. Not only 
$\mathrm{MOE}_{(/ /}$of $\mathrm{TP} m \mathrm{E}-\mathrm{H}$ was much higher than other groups, but also its $\mathrm{MOE}_{(/ /)}$was the largest. According to our previous study, the high $\alpha$-cellulose content contributed the higher MOE of TPmE-H [28]. It also indicated that $\mathrm{TP} m \mathrm{E}-\mathrm{H}$ contained higher holocellulose and $\alpha$-cellulose content than TMosoE-H; thus, the MOE value of TPmE-H was higher than TMoso-H. On the other hand, the OBSB made of bamboo with epidermis after SHT could increase $\mathrm{MOE}_{(/ /)}$, it is reasonable that the $\mathrm{MOE}_{(/ /)}$of $\mathrm{TPmE}-\mathrm{H}$ was higher than TMosoE-H. In addition to this, the anatomical characteristics in relation to the mechanical properties of bamboo culms also have been studied by several studies [1]. They concluded that vascular bundle size (radial/tangential ratio), distribution and concentration of vascular bundle and fiber dimensions (fiber length and wall thickness) correlated positively with MOE and stress at proportional limit. In this regard, it is worthwhile to further research and discuss the differences in the anatomical characteristics of the makino and moso bamboo to make a more comprehensive explanation. On the contrary, OBSB comprised bamboo strips after SHT and EPT showed the decline in $\mathrm{MOE}_{(/ /)}$. As shown in Table 2, the $\mathrm{MOE}_{(/ /)}$of the eight OBSB specimens ranged from 9.30 to $19.58 \mathrm{GPa}$, which was significantly higher than that of laminated bamboo boards made using Taiwan-origin moso bamboo (9.1 GPa) [9].

$\mathrm{MOE}_{(\perp)}$ of all eight OBSB specimens were much lower than their $\mathrm{MOE}_{(/ /)}$. Such difference is accounted for by the lower transverse strength of bamboo. Similar to the trend observed for $\mathrm{MOE}_{(/ /)}$, increase in $\mathrm{MOE}_{(\perp)}$ as a result of SHT was found only in OBSB comprising bamboo with epidermis while reduced $\mathrm{MOE}_{(\perp)}$ was observed in bamboo after SHT and EPT. As shown in Table 2, the $\mathrm{MOE}_{(\perp)}$ of the eight OBSB specimens ranged from 1.06 to $2.09 \mathrm{GPa}$, which was significantly lower than that of laminated bamboo boards made using Taiwan-origin moso bamboo (2.6 GPa) obtained by Lee et al. [9].

\section{Modulus of rupture (MOR)}

The results of $\mathrm{MOR}_{(/ /)}$(Table 2) were in general similar to those of $\mathrm{MOE}_{(/ /)}$. That is, the $\mathrm{MOR}_{(/ /)}$of OBSB comprised makino bamboo with epidermis intact were significantly higher than their moso bamboo counterparts from both Taiwan and China whether SHT or not. OBSB made of Taiwanorigin bamboo had distinctly higher $\mathrm{MOR}_{(/ /)}$than Chinaorigin samples. The $\mathrm{MOR}_{(/ /)}$difference between TMosoE and TMoso was insignificant $(p<0.05)$, indicating that EPT slightly effected in $\mathrm{MOR}_{(/ /)}$of moso bamboo. However, SHT significantly augments $\mathrm{MOR}_{(/ /)}$of unpeeled makino bamboo but decreases $\mathrm{MOR}_{(/ /)}$of moso bamboo with and without EPT. The OBSB bad of peeled moso bamboo and Chinaorigin bamboo were dramatically reduced their $\mathrm{MOR}_{(/ /)}$. Chung and Wang evaluated the impact of EPT and SHT on makino and moso bamboo, and attributed the variations in strength to their difference in holocellulose contents; a close correlation between holocellulose content and strength in both bamboo and wood [28]. Compared with the $\mathrm{MOR}_{(/ /)}$ of $95.6 \mathrm{MPa}$ for parallel-grain structural laminate boards made of Taiwan-origin moso bamboo reported by Lee et al., all eight OBSB specimens in this study revealed a higher $\mathrm{MOR}_{(/ /}$, with the minimum being $98.3 \mathrm{MPa}$ for CMoso-H and the maximum being 196.5 MPa for TPmE-H [9].

Furthermore, $\mathrm{MOR}_{(\perp)}$ of all eight OBSB specimens were much lower than their $\mathrm{MOE}_{(/ /)}$. As shown in Table 2, the $\mathrm{MOR}_{(\perp)}$ ranged from 2.28 to $12.4 \mathrm{MPa}$, which were significantly lower than that of laminated bamboo boards made using Taiwan-origin moso bamboo (15.8 MPa) obtained by Lee et al. [9]. The transverse strength of OBSB depends mainly on strength of the resin between bamboo strips, thus accounting for the lower $\operatorname{MOR}_{(\perp)}$ in OBSB specimens than the laminated bamboo board. SHT augments $\mathrm{MOR}_{(\perp)}$ of unpeeled makino bamboo and moso bamboo but decreased $\mathrm{MOR}_{(\perp)}$ of their peeled counterparts.

\section{Profile density distribution analysis}

Profile density distribution is an essential index for wood composite mechanical properties. Winistorfer et al. suggested that high-temperature compression caused the density of the outer layer to be significantly higher than that of the middle layer of wood composites [29]. Figure 3 shows the profile density distribution of different layers of OBSB specimens. There were variations in density among the layers, and the differences in density were significant $(p<0.05)$ for TP $m \mathrm{E}$ and TMosoE. On the contrary, OBSB specimens made of peeled moso bamboo showed smaller variations in density at different layers. That is to say, the differences in density observed in bamboo after EPT were insignificant $(p>0.05)$. Furthermore, comparing the density before and after SHT shows more uniform density at different layers of OBSB. It indicates that the differences in density between layers in steam-heated OBSB specimens become insignificant as a result of SHT $(p>0.05)$. Taken together, after EPT and SHT could reduce variations in density, these two processes increased more uniform profile densities in OBSB.

\section{Internal bond strength (IB) analysis}

Kojima and Suzuki used IB as the index for determined durability of various woody composites and reported a positive correlation between gluing strength and IB [30]. As shown in Fig. 4, the IB of OBSB specimens made of peeled bamboo, i.e., TMoso (3.04 MPa) and CMoso (2.83 MPa), were higher than the samples without EPT. It means that EPT increased IB strength in moso bamboo. However, SHT reduced IB of OBSB specimens made of peeled bamboo, 

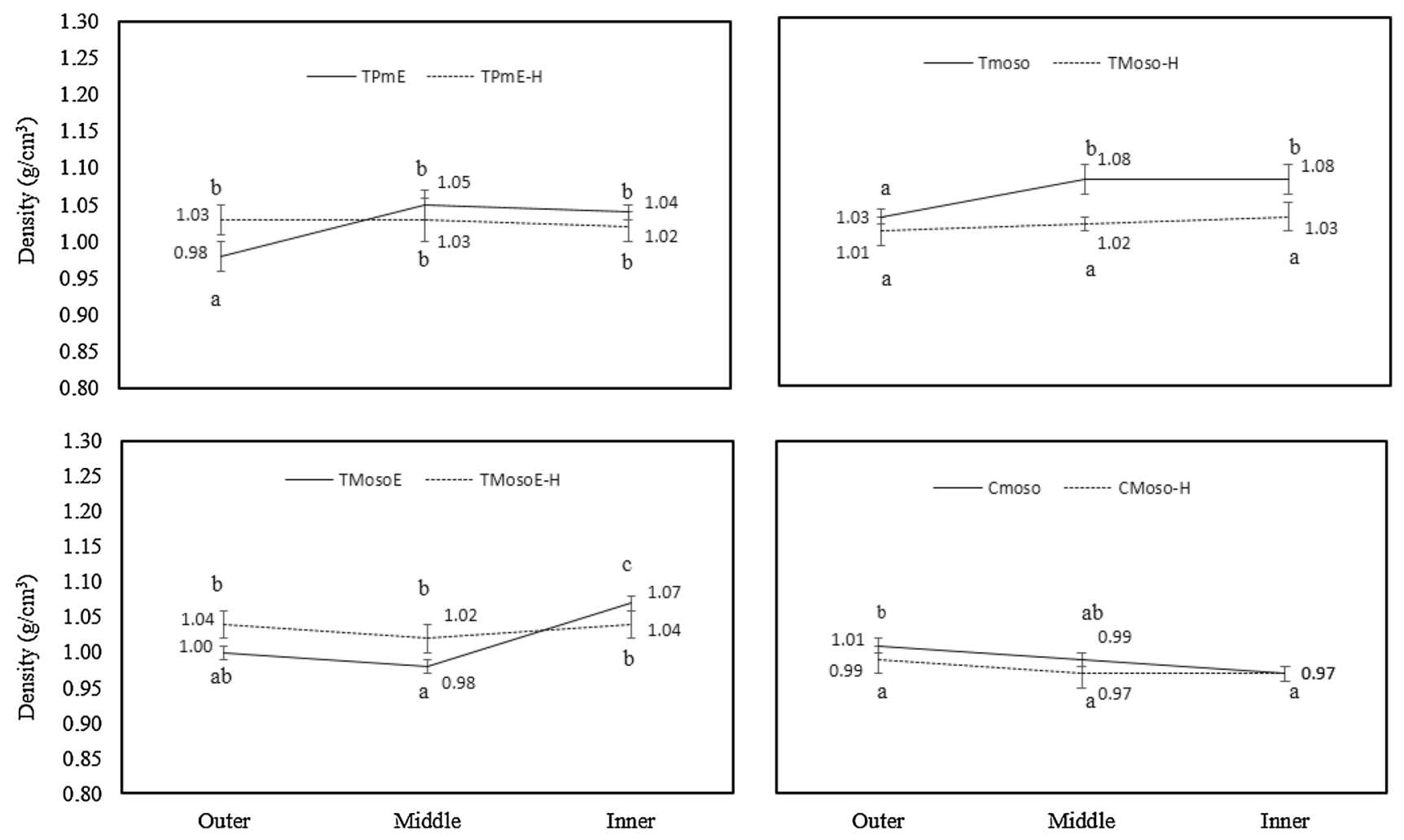

Fig. 3 Profile density of eight OBSB specimens. (Different letters indicate significant differences at 0.05 level obtained by ANOVA.)

Fig. 4 Internal bond strength (IB) strength of eight OBSB specimens. (Different letters indicate significant differences at 0.05 level obtained by Tukey's test and ANOVA.)

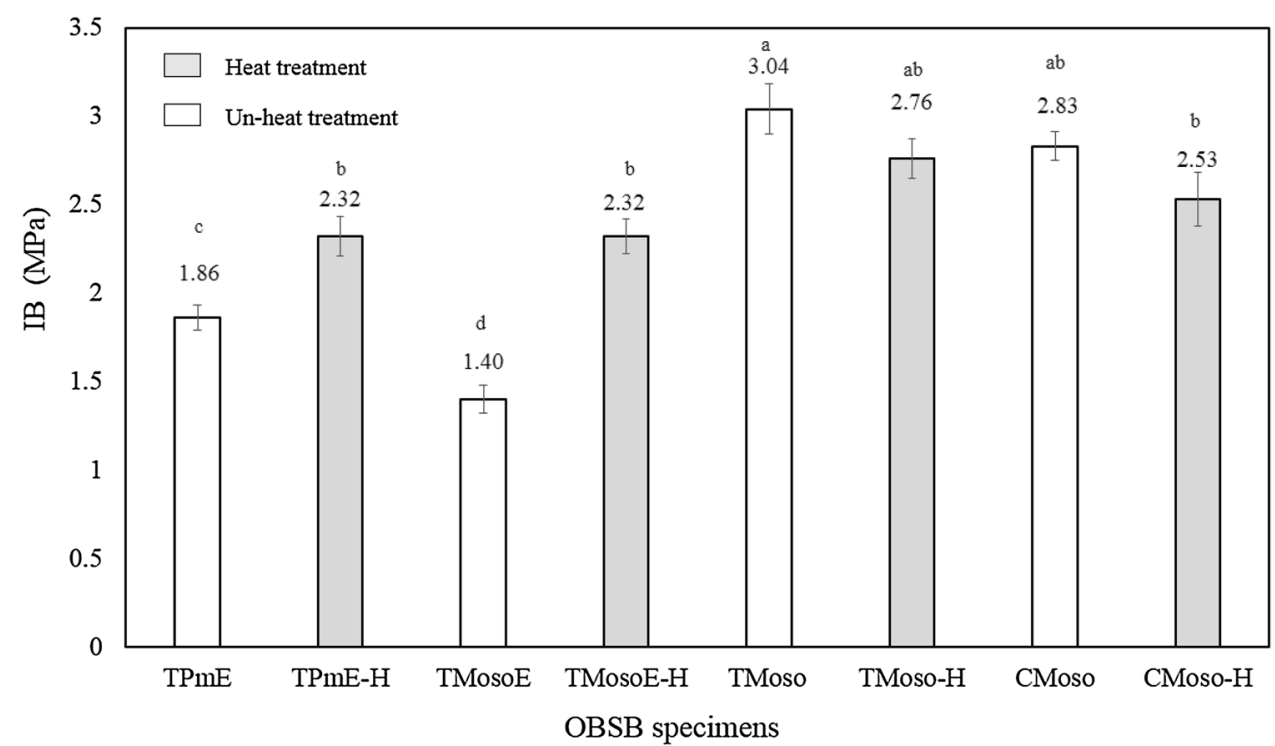

TMoso-H (2.76 MPa) and CMoso-H (2.53 MPa). In contrast, OBSB specimens made of steam-heated bamboo with epidermis showed increment in IB. SHT enhanced IB of without EPT bamboo. In the case of TPmE and TMosoE, their significant difference in IB (1.86 and $1.40 \mathrm{MPa}$, respectively, $p<0.05$ ) was eliminated by SHT, with their IB both increased to $2.32 \mathrm{MPa}$. In summary, OBSB specimens comprised bamboo strips without EPT had higher IB after SHT while such treatment will cause IB to reduce in OBSB specimens consisting of peeled bamboo strips. Lin and Huang reported the similar result [31]. Thermal compression enhanced gluing quality and further increase density for the particle board; thus strengthening the internal bond. In conclusion, the present results show that the IB of all eight 
OBSB specimens meets the standards for CNS 2215 [27] surface particle board (0.3 MPa) and EN 312 Europe cohesion strength (0.4 MPa) [32].

\section{Springback (SB) analysis}

Lee et al. evaluated the physical and mechanical properties of OSB made of moso bamboo and reported that resin content had significantly effect on MOE, MOR, IB and TS, but not on SB, linear expansion and nail withdrawal resistance [33]. As shown in Fig. 5, SB decreased after steam-heating treatment with the most significant reduction observed in $\mathrm{TP} m \mathrm{E}$, from 12.50 to $8.33 \%$. Moreover, OBSB made of Taiwan-origin makino and moso bamboo had lower SB than those comprising peeled Taiwan-origin and China-origin moso bamboo without EPT. Taken together, the results show that peeling treatment caused higher SB, while steam-heating reduced SB. Tabarsa and Chui explained lower density of woody composites with a lower SB value is due to the vessel and the cell lumen within the tissue of the bamboo, which are not compressed after hot pressing process [34]. Thus, it can be agreed with SB and density of OBSB specimens was a proportional relationship.

\section{Nail withdrawal resistance analysis}

As shown in Fig. 6, OBSB made of bamboo with SHT treatment, both with and without EPT, had higher nail withdrawal resistance. SHT enhanced the nail holding strength, with the most significant increment observed in OBSB made of China-origin moso bamboo. Moreover, Makino bamboo OBSB had lower nail withdrawal resistance compared with moso bamboo ones. Furthermore, OBSB comprising peeled moso bamboo strips had higher nail withdrawal resistance compared with their counterparts without EPT. EPT had a positive effect on nail holding strength. This result with the contents of benzene-alcohol extraction, hemicellulose of eight different bamboo [28] and the IB value of eight OBSB specimens analysis have similar trends. Therefore, it is speculated that since EPT and SHT will increase the content of benzene-alcohol extraction and hemicellulose in bamboo materials, it will contribute to produce more gluing areas and higher IB values during hot pressing. This result will help to enhance the mechanical interblocking effect of the OBSB board for wood screws. Indirectly improves the value of the nail withdrawal resistance. Besides, the nail withdrawal resistance of all eight OBSB specimens exceeded $51 \mathrm{kgf} / \mathrm{cm}^{2}$, the CNS 2215 standard on the particle boards of type 35-15 [27].

\section{Dimensional stability}

Previous research reported that heat treatment caused wood modification, thus reducing $\mathrm{S}$ and hygroscopicity $[35,36]$. As shown in Table 3, OBSB specimens comprising CMoso had the highest WA\% while those containing TPmE had the lowest WA\%. SHT bamboo strips resulted in significant decrease in WA\% of all OBSB specimens $(p<0.05)$. Mohebby and Llbeighi indicated that SHT caused degradation of hemicellulose, which affected the hygroscopicity of the woody material [37]. Moreover, fibers in the noncrystalline region of cellulose undergo increased crystallinity, which makes it retard water to enter the fiber. The same decrement in hygroscopicity of bamboo was also observed by Lee et al. [9].

Furthermore, SHT caused a significant reduction in both TS\% and S\% in all OBSB specimens, with the most significant decrease (TS \% from 16.2 to $4.82 \%$, S\% from 22.7 to
Fig. 5 Springback of eight OBSB specimens. (Different letters indicate significant differences at 0.05 level obtained by Tukey's test and ANOVA.)

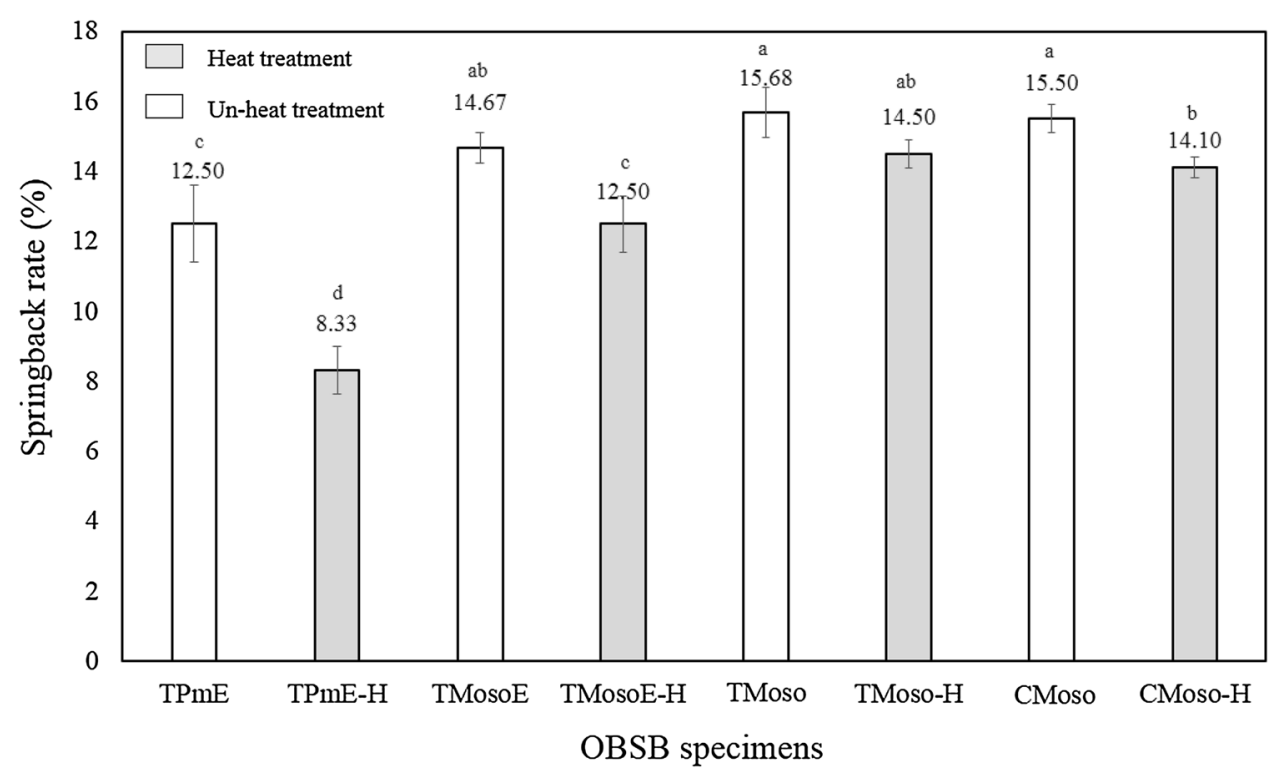


Fig. 6 Nail withdrawal resistance of eight OBSB specimens. (Different letters indicate significant differences at 0.05 level obtained by Tukey's test and ANOVA.)

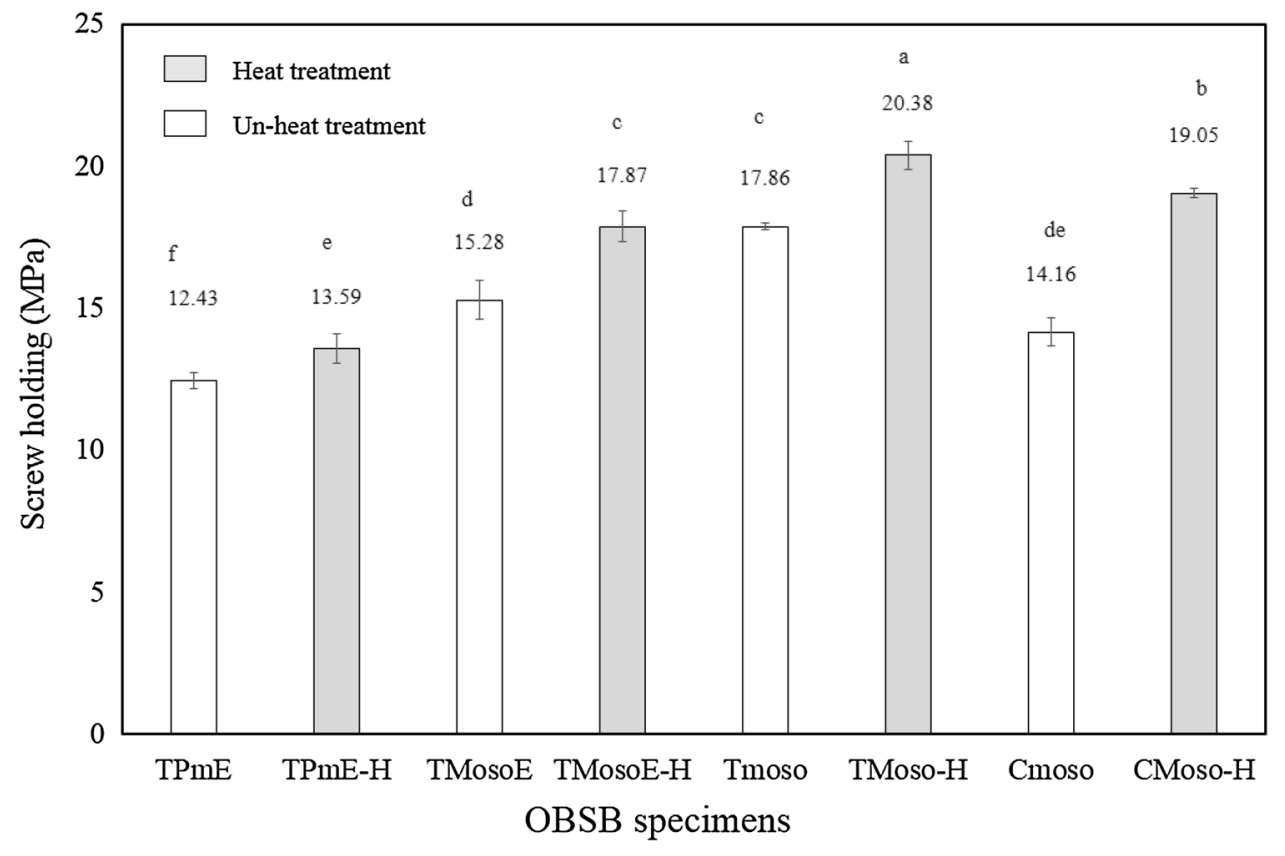

Table 3 Thickness swelling coefficient and volumetric swelling coefficient of eight oriented bamboo scrimber board (OBSB) specimens

\begin{tabular}{lllllr}
\hline $\begin{array}{l}\text { Board type \& } \\
\text { process }\end{array}$ & Code & WA (\%) & TS (\%) & S (\%) \\
\hline Taiwan & Makino & TPmE & $25.7(2.1)^{\mathrm{c}}$ & $13.7(1.7)$ & $24.0(0.9)$ \\
& & TPmE-H & $15.7(1.5)^{\mathrm{d}}$ & $7.98(0.6)$ & $12.6(0.9)$ \\
& \multirow{2}{*}{ Moso } & TMosoE & $26.0(1.0)^{\mathrm{c}}$ & $11.9(0.9)$ & $20.7(0.9)$ \\
& & TMosoE-H & $16.5(1.3)^{\mathrm{d}}$ & $6.99(2.0)$ & $10.5(2.7)$ \\
& & TMoso & $28.0(4.1)^{\mathrm{b}}$ & $10.2(1.8)$ & $15.6(2.7)$ \\
\multirow{3}{*}{ China } & Moso & TMoso-H & $12.2(1.5)^{\mathrm{e}}$ & $4.81(1.1)$ & $6.6(1.0)$ \\
& & CMoso & $31.0(4.3)^{\mathrm{a}}$ & $16.2(3.6)$ & $22.7(4.8)$ \\
& & CMoso-H & $15.5(1.1)^{\mathrm{de}}$ & $4.82(1.1)$ & $6.8(1.3)$ \\
\hline
\end{tabular}

Different letters a, b, c, d and e in a given column indicate significant differences at 0.05 level obtained by Tukey's test and analysis of variance (ANOVA)

$W A$ water absorption, $T S$ thickness swelling, $S$ volumetric swelling. Abbreviations for code are defined in Table 1

6.76\%) observed in OBSB comprised CMoso. These results prove that dimensional stability can be greatly improved in OBSB made of SHT bamboo strips. Kojima and Suzuki [30] pointed out that mechanical strength of composite boards is positively correlated with IB and inversely related to TS \%. Hence, reduction in TS \% as a result of SHT would also lead to strength enhancement.

\section{Conclusions}

Oriented bamboo scrimber board (OBSB) made of makino and moso bamboo strips with and without EPT and SHT showed different strength properties. Results obtained using NDT revealed that EPT applied to moso bamboo in the processing of OBSB led to lower $V_{\mathrm{u}(/ /)}$, higher $V_{\mathrm{u}\left({ }^{\perp}\right)}$, lower $\mathrm{DMOE}_{\mathrm{u}(/ /)}$ and higher $\mathrm{DMOE}_{\mathrm{u}(\perp)}$ while it affected less significant variations in MOE and MOR of moso bamboo. In contrast, the impact of SHT on $V_{\mathrm{u}}$ and $\mathrm{DMOE}_{\mathrm{u}}$ was inconsistent and insignificant among the OBSB specimens. On the other hand, SHT caused the increase in MOE and MOR of OBSB comprising bamboo with epidermis intact but led to decrease in MOE and MOR of OBSB contained peeled bamboo strips. Both EPT and SHT contributed to more uniform profile densities in OBSB and had a positive impact on nail withdrawal resistance. EPT increased IB of moso bamboo but SHT enhanced IB of bamboo with epidermis only. Bamboo strips after SHT resulted in significant decrease in WA of all OBSB specimens. Reduction in swelling as a result of SHT not only improved the dimensional stability of OBSB but also enhanced strength.

Acknowledgements This study was supported by a Grant (106-A03-5) from the Experimental Forest, College of Bioresource and Agriculture, National Taiwan University, Taiwan, ROC. We also thank the Forestry Bureau for financial support. 


\section{References}

1. Liese W (1987) Research on bamboo. Wood Sci Technol 21:189-209

2. Obataya E, Kitin P, Yamauchi H (2007) Bending characteristics of bamboo (Phyllosachys pubescens) with respect to its fiber-foam composite structure. Wood Sci Technol 41:385-400

3. Khalil HPSA, Bhat IUH, Jawaid M, Zaidon A, Hermawan D, Hadi YS (2012) Bamboo fibre reinforced biocomposites: a review. Mater Design 42:353-368

4. Lee AWC, Bai X, Bangi AP (1998) Selected properties of laboratory-made laminated bamboo lumber. Holzforschung 52:207-210

5. Lee AWC, Liu Y (2003) Selected physical properties of commercial bamboo flooring. Forest Prod J 53:23-26

6. Okubo K, Fujii T, Yamamoto Y (2004) Development of bamboobased polymer composites and their mechanical properties. Compos Part A-Appl S 35:377-383

7. Sulastiningsih IM, Nurwati (2009) Physical and mechanical properties of laminated bamboo board. J Trop Forest Sci 21:246-251

8. Verma CS, Chariar VM (2012) Development of layered laminate bamboo composite and their mechanical properties. Composites: Part B: Engineering 43:1063-1069

9. Lee CH, Chung MJ, Lin CH, Yang TH (2012) Effects of layered structure on the physical and mechanical properties of laminated moso bamboo (Phyllosachys edulis) flooring. Constr Build Mater 28:31-35

10. Yu Y, Zhu R, Wu B, Hu Y, Yu W (2015) Fabrication, material properties, and application of bamboo scrimber. Wood Sci Techno 49:83-98

11. Wang JX (1989) Bamboo scrimber: novel technology and novel product. China Wood Ind 3:52-53

12. Yu WJ, Yu YL (2013) Development and prospect of wood and bamboo scrimber industry in China. China Wood Ind 27:5-8

13. Sharma BA, Gatóo MB, Ramage M (2015) Engineered bamboo for structural applications. Constr Build Materi 81:66-73

14. Sharma B, Gatóo A, Ramage MH (2015) Effect of processing methods on the mechanical properties of engineered bamboo. Constr Build Mater 83:95-101

15. Nugroho N, Ando N (2001) Development of structural composite products made from bamboo II: fundamental properties of laminated bamboo lumber. J Wood Sci 47:237-242

16. Bekhta P, Niemz P (2003) Effect of high temperature on the change in color, dimensional stability and mechanical properties of spruce wood. Holzforschung 57:539-546

17. Esteves B, Pereira H (2009) Wood modification by heat-treatment: a review. BioResources 4:370-404

18. Chang ST, Chang HT (2001) Comparisons of the photostability of esterified wood. Polym Degrad Stab 71:261-266

19. Evans PD, Owen NL, Schmid S, Webster RD (2002) Weathering and photostability of benzoylated wood. Polym Degrad Stab 76:291-303

20. Borrega M, Kärenlampi PP (2008) Mechanical behavior of heattreated spruce (Picea abies) wood at constant moisture content and ambient humidity. Holz Roh Werkst 66:63-69
21. Zhao RJ, Jiang ZH, Hse CY, Shupe TF (2010) Effects of steam treatment on bending properties and chemical composition of moso bamboo (Phyllostachys pubescens). J Trop Forest Sci 22:197-201

22. Yildiz S, Gezer ED, Yildiz UC (2006) Mechanical and chemical behavior of spruce wood modified by heat. Build Environ 41:1762-1766

23. Mishiro A (1996) Effect of density on ultrasonic velocity in wood. Mokuzai Gakkaishi 42:887-894

24. Ross RJ, McDonald KA, Green DW, Schad KC (1997) Relationship between $\log$ and lumber modulus of elasticity. Forest Prod $\mathrm{J}$ 47:89-92

25. Lin CJ, Tsai MJ, Wang SY (2006) Nondestructive evaluation techniques for assessing dynamic modulus of elasticity of moso bamboo (Phyllosachys edulis) lamina. J Wood Sci 52:342-347

26. ASTM D-1037 (1999) Standard test method for evaluating properties of wood base fiber and particle panel materials. American Society Testing and Materials (ASTM), West Conshohocken, PA

27. CNS 2215 (2012) Particleboards. Chinese National Standards (CNS), Republic of China, R.O.C.

28. Chung MJ, Wang SY (2017) Effects of peeling and steam-heating treatment on basic properties of Phyllostachys makinoi and Phyllostachys pubescens culms. J Wood Sci 63:473-482

29. Winistorfer PM, Xli W, Wimmer R (1995) Application of a drill resistance technique for density profile measurement in wood composite panels. Forest Prod J 45:50-53

30. Kojima Y, Suzuki S (2011) Evaluating the durability of woodbased panels using internal bond strength results from accelerated aging treatments. J Wood Sci 57:7-13

31. Lin HC, Huang JC (2001) Apply fade effective image processing analysis technique to evaluate internal bond strength of particleboard. Taiwan J Forest Sci 23:77-86

32. EN 312 (2003) Particleboards-specifications. European Committee for Standardization, Brussels, pp 1-21

33. Lee AWC, Bai X, Peralta PN (1996) Physical and mechanical properties of strandboard made from moso bamboo. Forest Prod J 46:84-88

34. Tabarsa T, Chui YH (1997) Effects of hot-pressing on properties of white spruce. Forest Prod J 47:71-76

35. Gunduz G, Aydemir D (2009) Some physical properties of heattreated Hornbeam (Carpinus betulus L.) Wood. Dry Technol 27:714-720

36. Korkut S, Bektas Î (2008) The effects of heat treatment on physical properties of Uludag fir (Abies bornmuelleriana Mattf.) and Scots pine (Pinus sylvestris L.) wood. Forest Prod J 58:95-99

37. Mohebby B, Llbeighi F (2007) Physical and mechanical properties of hydrothermally modified medium density fiberboard (MDF). Proceedings of the International Panel Products Symposium. Cardiff, Wales, UK 